\title{
Layers of Models in Computer Simulations
}

\author{
Thomas Boyer-Kassem
}

Thomas Boyer-Kassem is at the Laboratoire d'Histoire des Sciences et de Philosophie-Archives Henri Poincaré, Université de Lorraine. Correspondence to: Laboratoire d'Histoire des Sciences et de Philosophie-Archives Henri Poincaré, Université de Lorraine, 91 avenue de la Libération, BP 454, F-54001 Nancy, France. E-mail: thomas.boyer@univ-lorraine.fr

I discuss here the definition of computer simulations, and more specifically the views of Humphreys, who considers that an object is simulated when a computer provides a solution to a computational model, which in turn represents the object of interest. I argue that Humphreys's concepts are not able to analyse fully successfully a case of contemporary simulation in physics, which is more complex than the examples considered so far in the philosophical literature. I therefore modify Humphreys's definition of simulation. I allow for several successive layers of computational models, and I discuss the relations that exist between these models, the computer, and the object under study. An aim of my proposal is to clarify the distinction between computational models and numerical methods, and to better understand the representational and the computational functions of models in simulations.

\section{Introduction}

A computer simulation can be defined as a particular way of implementing a computation so as to get numerical results about the evolution of an object. Simulation is often analysed with the help of the concept of (computational) model, which is derived from a theoretical model and lends itself to being implemented within a computer. ${ }^{1}$ By looking at a case study of simulations that are more complicated than the ones which have been considered so far in the philosophical literature, I want to draw attention to some complex use of models in simulations. Namely, I argue that there are cases in which it is possible to identify several layers of models between what is solved by the 
computer and what is under study. Although identifying a plurality of models in simulations is a popular theme (Hughes 1999; Winsberg 1999), the novelty of my proposal is to acknowledge that several computational models are used within one simulation. One computational model represents the object, whereas the others represent other idealized analogical objects.

In this article, I have three aims: first, to enrich the set of examples of simulations that are considered by philosophers, as others might gain new ideas from the case study I present. My second and main aim is to refine the concept of a computer simulation, so as to provide a fine-grained picture in the case usual analyses do not apply very well. A by-product of that is, thirdly, to understand better the representational and the computational functions of models in simulations, and to clarify the distinction between computational models and numerical methods.

For brevity, I shall limit myself to discussing Humphreys's conception of simulations, which I present in section 2-but my thesis could easily be extended to other conceptions. ${ }^{2}$ In section 3 , I present some simulations used today in contemporary physics. In section 4, I discuss whether Humphreys's definitions enable him to analyse successfully these simulations. Finally in section 5, I present my proposal and distinguish it from others.

\section{Humphreys's Definitions of Simulation and of Computational Model}

\subsection{Humphreys's Definitions}

What is a computer simulation, for instance a simulation of the movement of a planet? For Humphreys (2004), a full simulation of a behaviour or of a system has two parts. The first part is the core simulation: it is 'the temporal part of the computational process' (Humphreys 2004, 109), which computes the numerical values of the required data (for instance, the core simulation provides the successive positions of the moving planet). The second part is the representation of these results, for instance through a numerical array, a graph, or an animated image. The core part of the simulation is 
defined in the following way:

System $S$ provides a core simulation of an object or process $B$ just in case $S$ is a concrete computational device that produces, via a temporal process, solutions to a computational model ... that correctly represents $B$. (Humphreys 2004, 110)

Humphreys distinguishes three elements: a concrete system $S$ (typically a computer), a computational model (cf. its definition below), and an object, a process, or a behaviour $B$. A graphical presentation of this definition is proposed in Figure 1. The computational model $(\mathrm{CM})$ has a central role insofar as it is connected with the other two elements: it represents $B$, and $S$ provides solutions to it. In other words, the computer $S$ doesn't directly provide numerical results for the behaviour, and Humphreys identifies what he calls a CM in between. What is simulated need not be a real object from our world (like some planet), and Humphreys also leaves room for simulations of idealized models and their behaviours (like the mean magnetization of the Ising model).

\begin{tabular}{rl|}
\hline represents $\nwarrow$ & Object, process, behavior B \\
produces solutions to $\nwarrow \nwarrow$ & Computational model CM \\
& System S \\
\hline
\end{tabular}

Figure 1: Diagram of Humphreys' (2004) definition of a simulation.

A CM is defined through six components:

1. A computational template, often consisting of a differential equation or a set of such, together with the appropriate boundary or initial conditions types. ...

2. The construction assumptions.

3. The correction set.

4. An interpretation.

5. The initial justification of the template. 
6. An output representation, which can be a data array, a graphical display, a general solution in the form of a function, or a number of other forms.

(Humphreys 2004, 102-103)

A computational template is usually derived from a theoretical template-like Newton's second law or Schrödinger's equation — which describes only general constraints; when the various forces, masses, etc. are specified, and when the equation is computationally tractable, then one has a computational template. The construction assumptions and the interpretation provide an ontology, which "specifies the kind of objects represented in the template' (Humphreys 2004, 78). These objects are ideal ones, like a point mass or a boson. A CM has two functions, which come from its template: 'Templates have multiple functions. One of these functions is to represent the world; another is to facilitate calculations.' (Humphreys 2004, 95). This is echoed by the central position a CM occupies in Figure 1: the representation function links it with $B$, and the facilitation of the calculation - brought by the form of the template - enables the system $S$ to produce solutions to the CM. Note that the 'representation' of $B$ by the $\mathrm{CM}$ is done in two different ways: by the template itself (cf. the quotation above, or Humphreys 2004, 80) and by the well-named output representation (for instance, the curve that is finally plotted by the computer).

\subsection{On the Identity of a Computational Model}

On which conditions should one consider that a change in a CM defines a distinct CM? One may consider that the slightest change in the syntax of the template, or in the ontology of the model, defines another CM. On the contrary, one may consider that only major changes, like a change in the number of dimensions, defines a new $\mathrm{CM}$. Humphreys doesn't explicitly take a position, and I won't argue for precise identity criteria for CMs either, because I don't need them for the support of my thesis. What I need is only a lower limit: some agreement on the fact that, for the analysis of computer simulations, some changes are sufficiently important to define different CMs. Such a limit is needed because, as I will be arguing for the acknowledgement of several CMs instead of one, an objection could be that I identify new CMs too easily. A reader willing to 
consider that there are more different CMs that I allow for will be only more supportive of the claims I make in the subsequent sections.

What I have in mind as a 'sufficiently important' change is for example the reinterpretation of an equation to apply it to another domain of phenomena. To take an example from Humphreys, the Klein-Gordon equation, which was interpreted as being about 'a flexible string embedded in a thin sheet of rubber' can be reinterpreted as being about 'a relativistic spinless particle in a null electromagnetic field' (Humphreys 2004, 70). In these cases, the worlds depicted by the equation (its interpretations) and what the CMs possibly represent are clearly different. Given that the representation of the object and the interpretation of the model are central points in Humphreys's definition of a CM, it seems to be in line with Humphreys's view to consider that a reinterpretation of an equation is a sufficiently big change to define a new CM, even if the template is left unchanged.

Reciprocally, mathematical transformations of the template can also count as a sufficiently important difference in CMs. For instance, if a vector is projected onto a lower-dimension subspace, if complex quantities are transformed into real ones, if the number of space dimensions is changed, then I consider that a new CM should be acknowledged. Here, I should discuss specifically algorithms, subroutines, and other numerical methods, which might be said to alter a template. They do not appear in Humphreys's definition of a simulation, but he alludes to them elsewhere (Humphreys 2004, 81, 103-105, 112), and a definition can be reconstructed: they help to find approximate numerical values for the solutions to a given problem. Hence, Humphreys's definition of a simulation can be supplemented accordingly: 'a system $S$ provides a core simulation ... in case $S$ is a device that produces, via a temporal process and with the help of numerical methods, solutions to a CM'.

The use of a numerical method can bring changes in ontology. For instance, when a space parameter is discretized, the space ontology consists in points instead of a continuum. Yet, it still refers to space, not to something else (time, another planet...). So, the change in ontology exists here but is smaller than in the case of the reinterpretation of the full model that I have discussed above. Since my argumentative strategy needs to consider only sufficiently big changes, I choose for ease to discard this 
one, without needing to convince the reader that the change is worth a new $\mathrm{CM}^{3}$ So, if two CMs differ only by a numerical method, and in particular by the discretization of their parameters, I will consider that they are the same.

\section{A Case Study in Quantum Physics: ITPISR Simulations}

This section is dedicated to the presentation of a class of simulations, which are used in many contemporary fields of physics. These simulations involve what is called an 'imaginary time path integral', with a statistical reinterpretation of the equations, so I call them ITPISR simulations. They are very popular and have been widely used for a couple of decades. For pedagogical reasons, I introduce them through the example of a particular model, the quantum rotor.

\subsection{Overview of an ITPISR Simulation of the Quantum Rotor Model}

A quantum rotor consists in a particle of mass $M$, which can move on a circle of radius $R$ (cf. Figure 2). ${ }^{4}$ The position of the particle is denoted with the angle $\varphi$. The particle represents a rotor with a moment of inertia $I=M R^{2}$. Its Hamiltonian reads

$\hat{H}=-\frac{\hbar^{2}}{2 I} \frac{\partial^{2}}{\partial \varphi^{2}}$. The quantum rotor is a generic model, which can be used to model many different situations. It can represent a rotator whose axis is fixed, but also an electron moving in a periodic crystal or an electron winding around a closed space filled with a magnetic field (Aharonov-Bohm effect). A quantity that physicists are especially interested in simulating is the transition probability amplitude between two states. For instance, the amplitude for starting at the angle $\varphi=0$ at time $t=0$ and ending at the angle $\varphi_{T}$ at $T$ is written $\left\langle\varphi_{T}, T \mid 0,0\right\rangle$, and is also called the propagator $K\left(\varphi_{T}, T, 0,0\right)$.

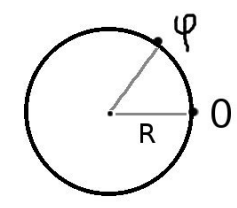

Figure 2: The quantum rotor.

In order to compute $K\left(\varphi_{T}, T, 0,0\right)$, one might solve Schrödinger's differential equation so as to obtain $\left|\varphi_{T}, T\right\rangle$ as a function of the initial condition $|0,0\rangle$ (similarly to 
solving Newton's second law in classical mechanics). Alternatively, $K$ can be computed with an equivalent formalism, called the path integral. ${ }^{5}$ This formalism relies on an integral over paths, which are functions $\varphi(t)$ defined on $[0, T]$. For $K\left(\varphi_{T}, T, 0,0\right)$, only paths with the right boundary conditions $\varphi(0)=0$ and $\varphi(T)=\varphi_{T}$ are considered. Each path receives the complex weight $e^{i S[\varphi(t)]}$, where $S$ is the classical action. Then, $K$ is given by the sum of this amplitude for all paths with the appropriate boundary conditions:

$$
\left.K\left(\varphi_{T}, T, 0,0\right)=\int_{0,0}^{\varphi_{T}, T} \mathcal{D}_{[\varphi]}\right]^{\frac{i}{\hbar} S[\varphi]} .
$$

Whereas the simulation of the Schrödinger differential equation computes the value of $\varphi(t)$ temporal step by temporal step, the path integral simulation considers every possible value, with appropriate weight. The path integral formalism looks computationally quite complicated, as the integration space has an infinite number of dimensions. But some tricks make the simulation actually much more convenient.

Given the path integral formulation, the first trick is to make a mathematical transformation, called switching to imaginary time $\left(t \mapsto i \times t\right.$, with $\left.i^{2}=-1\right)$. The formulation obtained is known as 'imaginary time path integral'. The amplitude for any path, which used to be a complex number, is now a real number. This speeds up very much the convergence of the final sum - the phase doesn't oscillate in the complex plane any more. After this mathematical transformation, the equations are reorganized to define new variables (called 'Euclidean'): for example, a new action $S_{\text {Euclidean }}$ and a new propagator $K_{\text {Euclidean }}$ are defined from the former $S$ and $K$.

In the Euclidean model, Euclidean time is discretized. In other words, $\varphi$ is defined only on some discrete Euclidean time-slices (say $L$ ) between 0 and $T$. Since Euclidean time is similar to space, these time-slices are usually considered as nodes on a lattice, and the discretized Euclidean model is known as the lattice model (for instance, the propagator $K_{\text {Euclidean }}$ is then called the lattice propagator $\left.K_{\text {lattice }}\right)$. On the lattice, the formerly continuous function $\varphi(t)$ of a path becomes a set of values $\varphi_{k}$ on each node $k=1, \ldots, L$ (cf. Figure 3$)$.

How can $K_{\text {lattice }}$ be simulated? To compute it on every path configuration, the 


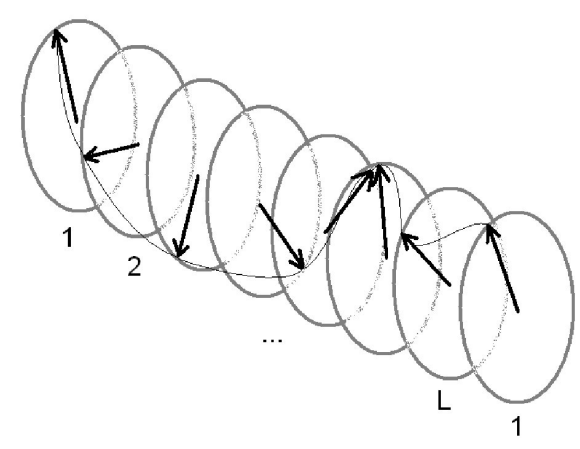

Figure 3: Example of a path on the lattice.

variable $\varphi$ could be discretized, but it would still demand huge computing resources. A second trick comes into play. ${ }^{6}$ It can be noticed that $K_{\text {lattice }}$ has the form of a partition function $Z$ from classical statistical mechanics. That is, the variables in $K_{\text {lattice }}$ can be reinterpreted as some quantities in statistical mechanics, in such a way as to define a consistent classical statistical model, the XY model. The latter is thus a formal analogue of the lattice model, in the same way as a model of an electrical circuit can be an analogue of a mechanical model. The XY model is a generic model of statistical physics (like the better-known Ising model), where classical spins in two dimensions $\vec{J}_{k}$ interact with their nearest neighbours according to the Hamiltonian $H_{\text {classical }}=\sum_{k=0}^{L-1} \vec{J}_{k} \vec{J}_{k+1}$. The spins are laid in one spatial dimension (which corresponds to the original temporal dimension) like on a chain, in some temperature bath.

This analogy becomes useful from a computational viewpoint when a very powerful simulation technique, originally used to simulate statistical systems, is employed: the Monte-Carlo method, which 'consists simply of integrating over a random sampling of points instead of over a regular array of points' (Metropolis et al. 1953, 1088). ${ }^{7}$ This method proves to converge quicker than non-random methods, and has been further improved by the Metropolis et al. (1953) algorithm.

To sum up, when a physicist simulates the propagator $K$ of a quantum rotor, she actually starts by writing a program that simulates a spin chain, within a classical statistical theory. Switching from the statistical model to the analogue lattice model, she reinterprets the numerical value of the partition function $Z$ of the spin chain as $K_{\text {lattice }}$. Finally, she undoes the imaginary time transformation, expresses the quantities of the original quantum rotor in terms of the lattice ones, and gets the value of $K$ from $K_{\text {lattice }}$. 
These transformations are actually embedded within the computer program.

\subsection{Several Models}

Several models can be distinguished in this simulation: a path integral quantum model, a Euclidean model, a lattice model, and a statistical model. ${ }^{8}$ The path integral quantum model considers a quantum rotor, with a mass, a quantum action, a time of evolution, and so forth. In this model, $K$ is formulated with a path integral. In the Euclidean model, there is no time any more but another space dimension instead, and the equation of the propagator is mathematically different (real instead of complex). In the lattice model, space is discretized, and it is meaningful to consider $L$ nodes on the lattice; the mathematical expression of $K$ is still different, with an integral of products. The statistical model, finally, describes a chain of classical XY spins, with a classical action, at some temperature - all new quantities compared to other models.

There are both important and more minor differences between these models. The Euclidean and the lattice models, for instance, differ only by a discretization of the space parameter. Other models involve very different ontologies: existence or not of a temporal dimension, or of a temperature, various numbers of space dimensions, different interactions between the spins, and so on. It cannot be emphasized enough that the path integral quantum model, the Euclidean model (or the lattice model) and the classical statistical models have really different ontologies - or, to put it another way, the interpretations of these models are really different. When interpreted ontologically seriously, they picture very different worlds, with unequal number of dimensions, populated with very different entities.

These models are related in various ways. The equations of the Euclidean model are obtained from the equation of the path integral quantum model through an imaginary time transformation, plus a reinterpretation of the variables. For instance, the temporal variable $t$ is reinterpreted as a spatial variable in the Euclidean or lattice model, and renamed as a length. The correspondence between the propagators is

$$
K=\int_{0,0}^{\varphi_{T}, T} \mathcal{D}_{[\varphi]} e^{\frac{i}{\hbar} S[\varphi]} \quad \longleftrightarrow \quad K_{\text {Euclidean }}=\int_{0,0}^{\varphi_{T}, T} \mathcal{D}_{[\varphi]} e^{-\frac{1}{\hbar} S_{\text {Euclidean }}[\varphi]}
$$


The correspondence can be continued with the lattice model, which is just a discretized version of the Euclidean model. Table 1 (first two columns) shows the correspondence between some quantum and lattice quantities.

\begin{tabular}{|c|c|c|}
\hline $\begin{array}{c}\text { Path integral quantum model } \\
\text { (quantum rotor) }\end{array}$ & $\begin{array}{c}\text { Lattice model } \\
\text { (Euclidean rotor) }\end{array}$ & $\begin{array}{c}\text { Classical statistical model } \\
\text { (XY, classical spins) }\end{array}$ \\
\hline propagator $K$ & Euclidean propagator $K_{\text {lattice }}$ & partition function $Z$ \\
moment of inertia $I$ & moment of inertia $I$ & $\hbar a$ \\
quantum action $S$ & action on the lattice $S_{\text {lattice }}$ & classical action $S_{\text {classical }}$ \\
time $T$ & length of the lattice & length of the chain \\
& number of nodes $L$ & \\
& $\ldots$ & \\
& number of spins $L$ \\
\hline
\end{tabular}

Table 1: Correspondence between quantities in the models.

Compare the equations of $K$ and of $Z$ in the lattice and statistical models:

$$
K_{\text {lattice }}=\int \prod_{k} \mathrm{~d} \varphi_{k} e^{-\frac{1}{\hbar} S_{\text {lattice }}\left(\varphi_{k}\right)} \quad \longleftrightarrow \quad Z=\int \prod_{k} \mathrm{~d} \varphi_{k} e^{-\frac{H_{\text {classical }}\left(\varphi_{k}\right)}{k_{B} T}}
$$

If a temperature $\widetilde{T}$ and a classical Hamiltonien $H_{\text {classical }}$ are defined so that (with $k_{B}$ Boltzmann's constant)

$$
\frac{S_{\text {lattice }}}{\hbar} \longleftrightarrow \frac{H_{\text {classical }}}{k_{B} \widetilde{T}}
$$

then the equations of $K_{\text {lattice }}$ and $Z$ are formally the same. There is here no mathematical transformation between the equations, but only a formal analogy. It provides a radical change in the ontology (cf. the last two columns of Table 1).

Figure 4 sums up the relation between the models. They are linked in a successive way: the object under study is presented in the path integral quantum model, then it is changed into the Euclidean model, then the lattice model, and finally the statistical one. This is different from having four models which each represent different parts of a same system.

These models play an important role for physicists, who use the representations and the ontologies of the Euclidean, lattice and statistical models, not only of the quantum model. For example, Alet and Sørensen quickly adopt a 'link-current representation' (Alet and Sørensen 2003, 1, my emphasis), and talk only about the size of the lattice, its 


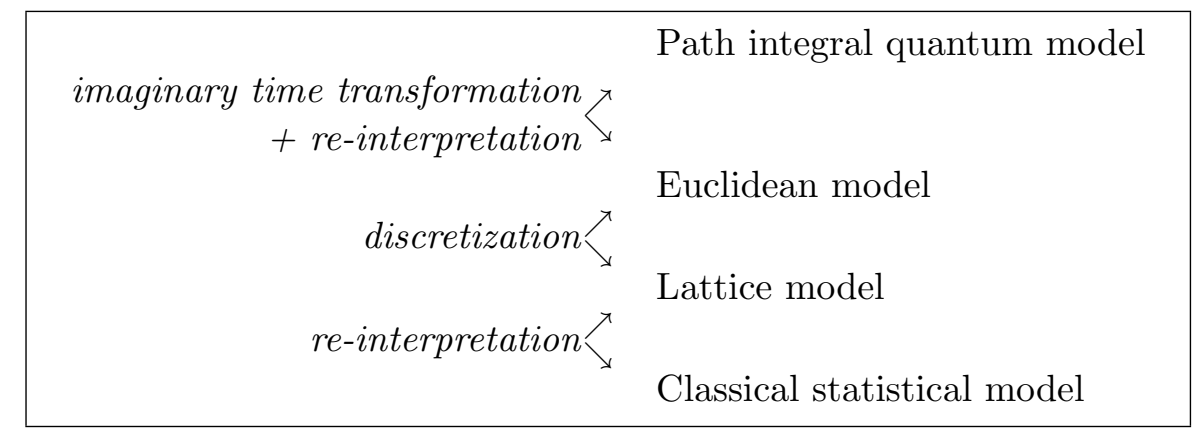

Figure 4: Links between the models.

sites, its links and the current on these links; they don't use the quantum representation any more. ${ }^{9}$ Boyer, Bietenholz, and Wuilloud (2007) also quickly switch to the statistical representation, talking about (classical) spins, XY model, and correlation length. When they define and study new objects (called 'clusters'), they do it first in the statistical model, not in the quantum one. Bietenholz et al. (1997) discuss approximations for simulation within the lattice (or Euclidean) model; the title of the paper itself contains the term 'lattice'.

These models also play a role in scientists' understanding, for instance in the sense proposed by De Regt and Dieks: 'a phenomenon $P$ is understood if a theory $T$ of $P$ exists which is intelligible', where the intelligibility of the theory is characterized by the ability of scientists to 'recognise qualitatively characteristic consequences of $T$ without performing exact calculations' (De Regt and Dieks 2005, 150, 151). This is the case for our simulation, and the above examples of use of the lattice and statistical models can be taken as cases of understanding in De Regt and Dieks's sense. For instance, saying that the statistical model helps a scientist to understand a quantum behaviour means that she can recognise a qualitative characteristic about the quantum behaviour if she directly uses the statistical theory on the XY spin chain. Indeed, it can be easier to reason about a chain of interacting spins than about complex superpositions of quantum paths.

\subsection{General Case}

The ITPISR way of simulating is not limited to the case of the propagator of the quantum rotor. Any behaviour or quantity of any quantum model can benefit from it: start with the quantum behaviour to be simulated, express it with a path integral 
formulation, perform the imaginary time transformation, reinterpret the model with Euclidean quantities, discretize time so as to get a lattice formulation, find the classical statistical model which is analogous to it, use a Monte-Carlo technique to get statistical averages, and then make the correspondence back up to the quantum behaviour.

ITPISR simulations can also be used outside the field of non-relativistic quantum mechanics, and for instance in its relativistic extension, quantum field theory. ${ }^{10}$ Here also, the main computational interest of ITPISR lies in the advantages provided by the statistical reinterpretation, and the Monte Carlo methods it enables - 'the entire book is based on this simple observation', says a textbook (Rothe 2005, 20). An additional motivation in using ITPISR simulations is that 'the lattice formulation provides the only possible framework at present to study QCD [a particular quantum field theory] non-perturbatively' (Rothe 2005, 3). Indeed, the results of ITPISR simulations are not limited in accuracy as perturbative techniques are, and can be improved simply by increasing the computational resource (Gupta 1999 discusses other motivations). Lattice quantum field theory (which contains the lattice models of quantum field theory) has become a major reformulation of quantum field theory: it has its own textbooks (like Montvay and Münster 1994, Gupta 1998, and Rothe 2005), and its own folder 'hep-lat' (for 'high-energy physics on the lattice') in the arXiv server for preprints.

String theory and general relativity also have a lattice formulation of the theory, and make use of ITPISR simulations. Thus, ITPISR simulations are essential ingredients for many contemporary physical theories, and are a scientifically important kind of simulation.

\section{Applying Humphreys's Analysis to ITPISR Simulations}

Since Humphreys's analysis intends to apply to all simulations, it should be able to analyse fruitfully ITPISR simulations in particular. That is, one should be able to identify a CM, within a core and a full simulation, that meets Humphreys's definitions. In this section, I argue that this can be done, but in a way that is not really illuminating. In the next section, I argue for a slight modification of Humphreys's definitions, so that ITPISR simulations can be analysed much more straightforwardly. 
The key concept in Humphreys's analysis is the CM. It would be rather easy to identify a CM around each of the 'models' of section 3.2: the path integral quantum, the Euclidean, the lattice and the classical statistical models. Consider the components. ${ }^{11}$ First, the template of the CM would be given by the form of the computed quantity, $K$ or $Z$. For example, in the case of the path integral quantum model, the template is the path integral formulation of the propagator $K=\int_{0,0}^{\varphi_{T}, T} \mathcal{D}_{[\varphi]} e^{\frac{i}{\hbar} S[\varphi]}$; for the statistical model, the template is the partition function $Z=\int \prod_{k} \mathrm{~d} \varphi_{k} \exp \left[-H\left(\varphi_{k}\right) / k_{B} \widetilde{T}\right]$. Second, consider what the interpretation of the $\mathrm{CM}$ would be. It would just be the interpretation of the model identified in section 3.2: for the path integral quantum CM, the ontology would consist in a mass, a quantum action, a time of evolution, etc. For the Euclidean $\mathrm{CM}$, there would be no time any more but another space dimension instead. For the lattice CM, the world would consist in a discretized space, with $L$ nodes on the lattice. For the statistical CM, the ontology would be a chain of classical XY spins, with a classical action, at some temperature. Finally, note that the Euclidean and lattice CMs thus defined differ only by a discretization of the space parameter. In accordance with section 2.2, I consider that this difference doesn't define two distinct CMs, and I will equivalently talk of the Euclidean or of the lattice CM.

To sum up, if CMs are identified around the models of an ITPISR simulation, there could be more or less three CMs: a quantum path integral CM, a lattice CM, and a statistical CM. Nevertheless, having identified these CMs is both too much and not enough. It is too much, because Humphreys's definition of a simulation allows for one $\mathrm{CM}$ in a simulation. And it is not enough (as such) because it must be shown also that this $\mathrm{CM}$ is in the right relationship with the other elements (cf. section 2.1, e.g. Fig. 1):

(i) the CM represents the object or the behaviour under study,

(ii) the CM is provided a solution by the computer.

So, some work still needs to be done to apply Humphreys's concepts to ITPISR simulations. I now consider several ways in which Humphreys's analysis cannot be applied, before discussing a way it can.

Consider the worry that there must be only one CM. Cannot one broad and big CM, instead of three, be identified? No, because of an incompatibility between the quantum 
template, with imaginary arguments, and the lattice (or statistical) template, with real arguments. No, also because no consistent interpretation could be defined: the quantum, the lattice, and the statistical models assume very different ontologies (cf. section 3.2).

Could it be argued that an ITPISR simulation actually involves several simulations, each with one CM? Thus, there would be three successive simulations: with the statistical CM, with the lattice CM, and finally with the quantum CM. The problem with this proposal lies in the very fact that an ITPISR simulation would consist in several successive simulations. Indeed, there is actually just one - albeit complex-simulation, where the computer program is run just once. For instance, there is no simulation using the lattice model: when the computer provides numerical solutions to the statistical model, no more computation needs to be done to get the numerical results for the lattice model. Only a reinterpretation of the parameters and a unit change are required, and this is nothing like having a computer run another simulation, and produce, via a temporal process, solutions to another CM. No simulation either is needed so as to get the quantum results from the lattice ones, but only an imaginary time transformation. ${ }^{12}$

Perhaps could it be argued that the quantum path integral $\mathrm{CM}$ is the unique $\mathrm{CM}$ of the simulation? In favour of this position is the fact that this CM actually represents the quantum rotor through its output representation, thus meeting the above requirement (i). What about requirement (ii), stating that the computer provides solutions to the CM? This is not exactly the case: numerical solutions are first solutions to the statistical CM (the Monte-Carlo method provides numerical results about the partition function of the spin chain). There are many steps before these solutions can become solutions of the quantum CM: the statistical reinterpretation, the imaginary time transformation, and reinterpretation. According to Humphreys's implicit position I have recalled in section 2.2, one has to say that these steps are numerical methods.

This leads to several problems. First, I have insisted in section 3.2 on the fact that the Euclidean, lattice or statistical models bring new representations and ontologies, which are used by physicists. Yet, what we usually mean by a numerical method is not supposed to bring new representations or ontologies (cf. section 2.2). The variables are supposed to refer to the same entities as the original model. For instance, if a model is interpreted with time and space variables, numerical methods are supposed to handle the 
same variables of time and space or, at worst, discretized ones. The Runge-Kutta algorithm, which is a typical numerical method used to solve differential equations, does not reinterpret the variables with different physical quantities, like time becoming space. Furthermore, numerical methods are not supposed to define new theories or models. For instance, the Runge-Kutta algorithm is independent of any theory and doesn't introduce any new theory. In our case, the so-called numerical methods actually rely on new theoretical models: the Euclidean model belongs to the Euclidean (or, when discretized, the lattice) quantum field theory, which has its own textbooks (cf. section 3.3). ${ }^{13}$ Even more clearly, the statistical model belongs to the classical statistical theory, which has nothing to do with the quantum theory. Reinterpreting a model thanks to an analogy is not what we would like to call an algorithm or a numerical method. As the imaginary time transformation and the reinterpretation lead to considering new theoretical constructs, they cannot be just considered as numerical methods, and the proposal has to be abandoned.

Let's consider a last way in which Humphreys's analysis could be applied to an ITPISR simulation: the statistical CM would be the single CM of the simulation. The advantage of such a position is that the requirement (ii) is now met: it is true that the computer provides solutions to the statistical model, and there is no problem of false numerical methods any more. With this proposal, the reinterpretation of the model into the lattice framework and the imaginary time transformation have to be considered as some post-treatment, after results are provided to the statistical CM. ${ }^{14}$ Humphreys's analysis would account for our case study, at the price of adding such a post-treatment. But is the requirement (i) satisfied, that is, is it true that the statistical CM represents the quantum rotor?

If the statistical CM represents the quantum rotor, it means that a model with an ontology of $L$ spins arranged on a line, interacting classically in some temperature bath, represents a quantum rotor freely moving on a circle with some inertia (more specifically, the length of the chain of spins represents time, the temperature represents the inverse of the inertia, and so on; cf. Table 1). Actually, this position is similar to arguing that, when there is an analogy between models in two fields of physics, a model from one field can be said to represent a phenomenon from the other. ${ }^{15}$ For instance, there is a 
well-known analogy between a swinging pendulum and an oscillating electric circuit, and so a model of an electric circuit can be said to represent a small object attached to a string. When such an analogical model is used, its ontology is very different from the phenomenon under study (or, one could say, from the ontology of the 'model of the phenomenon' one would make of it). In the above example, the ontology of the electric circuit is populated with electric currents, potential differences, resistances, capacitances, inductances and so on, whereas the ontology corresponding to the phenomenon under study consists in a point-like mass, a massless string, a gravity field, and so on. So, there is an intuitive sense in which an analogical model represents from farther the object of study, as the ontology which is used has primarily nothing to do with it.

Yet, I don't want to push further on that line, and I admit that Humphreys's concepts can be applied to ITPISR simulations. I acknowledge that the statistical CM can be said to represent the quantum rotor, even if indirectly as analogical model do (and for instance, more indirectly than it could represent a chain of atoms which interact with the classical XY action).

But I now want to argue that this application is not totally successful, in the sense that it is not detailed enough and misses important elements that it ought to capture. With this view of the statistical CM being the CM of the ITPISR simulation, the lattice and quantum models are totally kept silent - they are hidden in the large indirect analogous representation relation. This is a problem for at least three reasons. First, two other CMs can be identified on the neglected models (cf. the beginning of this section). Since a CM is a key concept for Humphreys, isn’t it a pity to have a definition of simulations with only one $\mathrm{CM}$, and that is not able to identify several CMs where it could be the case? Second, these two other CMs have representational roles: they define new ontologies and new fictional objects, which can be used for understanding, and they belong to other theories that are very important in physics (cf. section 3.2). Shouldn't an analysis of computer simulations point at what is representationally important and new? Finally, and more importantly, these CMs have very important computational roles: after the imaginary time transformation, the convergence of the integral in the Euclidean model is speeded up very much; lattice formulations are in some cases the only way to compute quantities non-perturbatively. Isn’t it the very function of a proper 
analysis of simulations to be able to point at the elements which improve the computation very substantially? In other words, my worry is that Humphreys's analysis of ITPISR simulations with a single statistical CM doesn't do justice to the two models (or CMs), quantum and lattice, which play crucial representational and computational roles in the simulation. It is a pity to hide the most interesting elements in a too broad 'analogical representation' relation.

But perhaps the best way to argue that Humphreys's analysis is incomplete is to present my own definitions in order to show that simulations, and ITPISR simulations in particular, can be analysed more successfully. This is done in the next section.

\section{Generalizing Humphreys's Analysis: Layers of Computational Models}

The previous section has argued that, although Humphreys's concepts can apply to ITPISR simulations, a finer-grained account would be very valuable. I offer here such an analysis.

\subsection{A Proposal}

I propose to keep Humphreys's definition of a CM, and to modify his definition of a computer simulation by allowing for several successive layers of CMs (my addition is in italics):

System $S$ provides a core simulation of an object or process $B$ just in case $S$ is a concrete computational device that produces, via a temporal process, solutions to a computational model that may be related by analogy or mathematical transformations successively to other computational models, where the last one of these layers correctly represents $B$.

My definition of a simulation is a generalisation of Humphreys's, since his corresponds to the case of one layer of CMs. So, my proposal keeps the benefit of Humphreys's view in this case. Note that the CMs are arranged successively, from the concrete device (the computer) to the object of interest $B$. The first layer of $\mathrm{CM}$ receives 
solutions from the computer, and the last one represents $B .{ }^{16}$ But these two parts are not necessarily played by the same CM. A CM on the layer $k>1$ receives solutions not directly from the computer, but from the CM on the layer $k-1$.

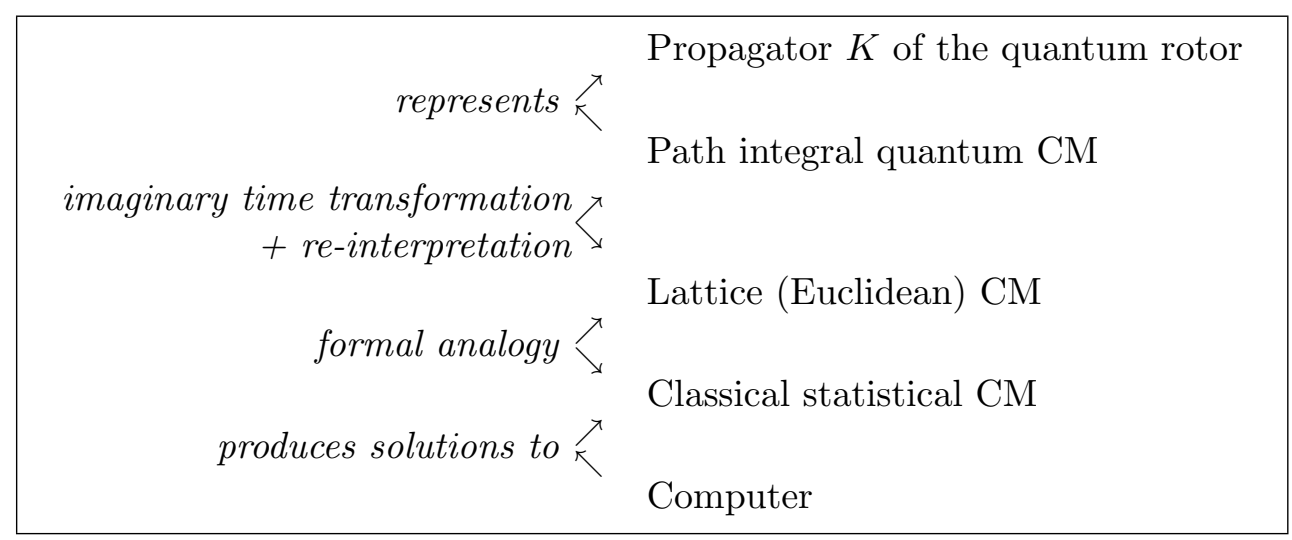

Figure 5: With my proposal, an ITPISR simulation is analyzed with three layers of CMs (to be compared with fig. 1 and 4 ).

How is an ITPISR simulation analysed with this new definition? My account leaves room for three CMs: the path integral quantum CM, the Euclidean or lattice CM, and the statistical CM, which are organized in layers as shown in Figure 5. Are these CMs each successively implemented in the computer program, and if not, to what extent do they exist? My position is, like Humphreys's, a reconstruction analysis: I want to identify the elements that justify thinking, from a theoretical viewpoint, that the kind of computation made by the computer is indeed a simulation of the object - for instance, to answer the question: why on Earth should one simulate an XY spin chain to get numbers about a quantum rotor? So, I do not claim that the layers of CMs are implemented one after the other in the program but that, to answer the above question, one needs to resort to the three CMs and to the analogies and to the mathematical transformations that relate them. It remains true that a single computer run solves the sequence of the three CMs, and it can be considered as a single simulation.

Why acknowledge several CMs in layers? I can see several reasons for that, which come in part from Humphreys's view itself: 'transformations of representations are commonly used in theories to facilitate computation, but many of these take us away from the realm of reality into the realm of the fictional' (Humphreys 2004, 96). In the case of ITPISR simulations, this sentence sounds particularly right: the initial quantum 
representation is successively transformed into lattice and statistical representations, and these models intend to represent not primarily just the quantum rotor, but rather other objects, like XY spins on a chain with a temperature bath, etc. In this sense, we can say that the lattice and statistical CMs represent (primarily) fictional objects like spins on a chain, whereas the quantum CM represents the quantum rotor. My proposal amounts to taking full act of the transformations of representations and the fictional objects they picture.

Recall also that, in an already quoted passage, Humphreys insists on the representation and the computational roles of CMs: 'Templates have multiple functions. One of these functions is to represent the world; another is to facilitate calculations.' (Humphreys 2004, 95). Indeed, the two supplementary CMs I identify have these representational and computational functions. The various models are used to represent different worlds populated with different ontologies (cf. section 3.2). Each template also has computational advantages (cf. section 4 ). So, why not explicitly identify templates (and around them, CMs) that have the very properties advocated by Humphreys? Thus, my proposal satisfies the complaint of section 4 that the lattice and quantum CMs were not acknowledged. My proposal also gives more sense to the fact that a quantum CM (and not a statistical $\mathrm{CM}$ ) represents the quantum rotor, because the ontology of the quantum $\mathrm{CM}$ is now much closer to the quantum rotor than the ontology of the statistical CM.

A last point in favour of the acknowledgement of the three CMs is that each of them can be independently reused as a CM, in Humphreys's original sense. That is, for each of the three candidate CMs, there exists a simulation with a single CM, in which the candidate $\mathrm{CM}$ is used. I can see three justifications for this criterion of independent reuse: first, it rules out cases where two CMs would always be found together in simulations, so that the relevance of distinguishing between them could be rightly questioned. It also prevents us from considering a numerical method as a CM, since numerical methods are always found attached to other CMs and are not used on their own. Finally, if each of the candidate CM is considered to be a CM in Humphreys's original sense when it is the only CM in a simulation, then it should also be considered as a CM in another simulation in which it is used among other CMs. Here, do the three 
CMs meet this independent reuse criterion? First, the path integral of the quantum propagator can be simulated with a crude discretization of the parameters (without imaginary time transformation or statistical tricks). In this case, the computer provides solutions directly to this CM, which represents the quantum propagator. Second, the lattice CM can also be used alone in a simulation of some lattice object, with a mere discretization of the parameters and without the statistical reinterpretation, in such a way that the computer directly provides solutions to this lattice CM. Finally, the case of a simulation involving only the statistical CM is straightforward, because it is what statistical physicists would do with any statistical model. Hence, the three candidate CMs meet the independent reuse criterion.

Let us now summarize the conditions on which several CMs can be identified in a multi-layered fashion. After identifying several models within the simulation, the following conditions should be checked:

1. The candidate model meets the conditions for being a CM (cf. above):

(a) it has a new representational function, which is used;

(b) it has a new computational function;

(c) it can be used alone in a possible simulation.

Condition (a) requires that the entities to which the model refers are different from the ones to which other models refer. A change in the number of spatiotemporal dimensions, the introduction or the suppression of a physical quantity are suitable differences in representation; a discretization of the parameters is not (cf. section 2.2). Furthermore, the new representation must actually be used by physicists, for instance in their qualitative reasoning (cf. section 3.2). ${ }^{17}$

2. The CMs are related as layers (like in Fig. 5): one CM receives solutions from the computer, another one represents the behaviour. For instance, this excludes simulations where different parts of the program model different aspects of the behaviour under study.

A by-product of these criteria for identifying a CM is that it provides a (negative) definition of numerical methods. They are what helps computation, but do not satisfy 
the criteria of being a CM. In particular, it is very likely that numerical methods do not bring a new representation. Conversely, when a numerical method has a representational role, and can be used alone in a simulation (as a CM), it is not a numerical method anymore, but a CM within a multi-layered simulation. In the case study of the simulation of the quantum rotor, the numerical method is only the Monte-Carlo method with the Metropolis algorithm.

\subsection{The Novelty of the Multi-layered Simulation Analysis}

Is my proposal of analysing simulations with several CMs new? I now consider several views which could claim to have already stated it.

First, consider a complex phenomenon which is represented with different models, one for each part of the phenomenon (for instance, a climate model has a sub-model for the ocean, another for the clouds, etc.). Haven't philosophers acknowledged such simulations with several CMs? But this has nothing to do with my proposal. Only one CM should represent the behaviour under study (condition 2), while others should represent other entities (condition 1a). Here, all the models represent some aspect of the same phenomena, and don't picture different worlds. In my analysis, the CMs are arranged in layers between the computer and the phenomenon, whereas the case described here would resemble more a horizontal plurality.

Consider now Hughes's analysis of computer simulations:

We may choose to remodel the original representation [of the phenomena] using a computer, in other words, to nest one representation within another in the manner shown in Figure [6]. The result is a hierarchy of models [in which] the whole of the second stage of the modelling process (enclosed within the dotted line in the diagram, and labelled 'Model 2') is performed on a computer. ... For this reason, we may deny that the simulation directly represents the phenomenon. (Hughes 1999, 131-132)

The 'DDI' account in Hughes's caption refers to his 'Denotion, Demonstration and Interpretation' account of scientific modelling (Hughes, 1997 and 1999, 124-128): elements of the phenomenon are denoted by elements of the model; the dynamics of the 


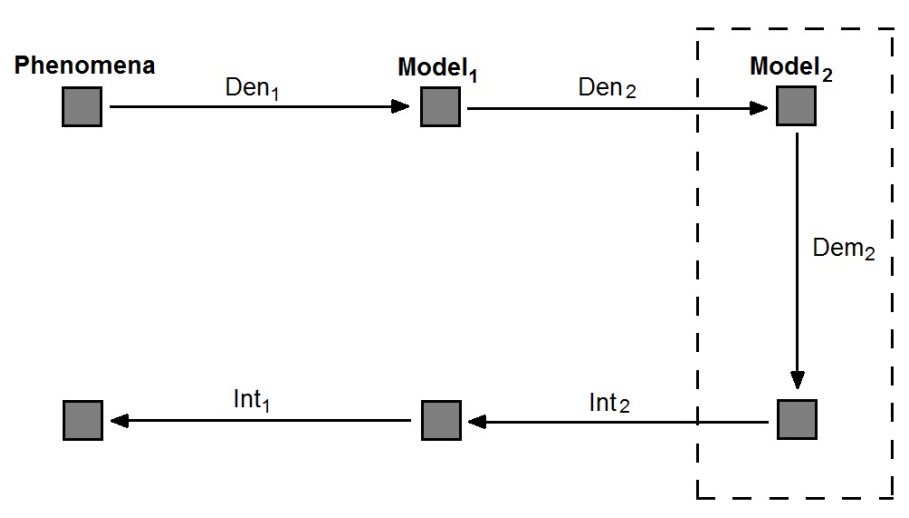

Figure 6: Hierarchies of representation on the DDI account of modelling [reproduced from Hughes $(1999,131)]$.

model enables us to demonstrate conclusions within the model, which can be interpreted back in terms of the phenomenon.

Doesn't my proposal amount to Hughes's analysis? Aren't there layers of models, only one directly representing the phenomenon? Actually, Hughes is adding models only outside the simulation (outside the dotted line of his diagram). Contrarily to my proposal, Model 1 and Model 2 are not computational models, used in the computer program. Model 1 is explicitly about describing the phenomenon in the terms of the theory. Hughes is still placing one model inside the computer (his Model 2); and the three CMs that I distinguish are meant to replace this single Model 2. Taking the viewpoint of section 5.1, one easily checks that Model 1 doesn't satisfy the conditions 1a, 1b or 1c. If Hughes had anticipated my proposal, the dotted line would encompass Model 1 and Model 2, and the relation between Model 1 and Model 2 would not be 'denotation' or 'interpretation', but 'formal analogy' or 'mathematical transformation'.

Consider now Winsberg, who insists on a hierarchy of models:

The integral part of the practice of simulation is the process of building what I call a hierarchy of these models. This hierarchy includes a mechanical model, a dynamic model, ad hoc models, a computational model, and finally, a model of the phenomena. ... Figure [7] illustrates the different layers of models involved in simulation and the resources used in each inferential step. (Winsberg 1999, 3-4)

Doesn't Winsberg advocate a picture that is similar to my proposal, with layers of 


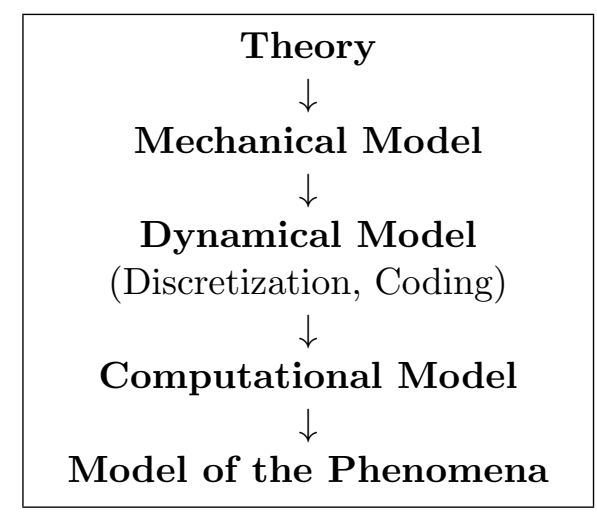

Figure 7: The Hierarchy of Models [reproduced from Winsberg $(1999,27)]$.

models? Note that Winsberg (1999, 4 and 7) claims to be doing 'applied epistemology': his analysis is about 'the practice of simulation', about how to justify the building of the various models from the theory. Thus, his hierarchy of models represents diachronic steps (a 'process', Winsberg 1999, 4). This is not a reconstruction analysis of a simulation considered as a finished product, studied from a synchronic viewpoint as Humphreys and I are doing. When Winsberg writes, 'once the computational model is implemented on a computer' (Winsberg, 1999, 10, my emphasis), he is acknowledging that only this model is implemented; other models of the hierarchy are not part of the simulation (they are just preparatory or justificatory steps for it). It is easy to see that, in all the models from Winsberg's hierarchy, none but the computational model satisfies the conditions 1a, 1b, 1c or 2. Actually, Winsberg agrees with Humphreys that a simulation consists in the implementation of a $\mathrm{CM}$. And nowhere does he consider the possibility that the $\mathrm{CM}$ might in fact be several CMs. My proposal is a departure as much from Humphreys as from Winsberg.

\section{Conclusion}

According to my proposal, some computer simulations are analysed with a single CM, while others require several CMs arranged in layers. For a given phenomenon, why is it that the simulation is done with several CMs, or simply with one? It seems to me that the reason is to be found in a conflict between the two functions Humphreys has rightly identified in templates (or CMs), representation and computation: 
The functions of accurate representation and ease of calculation are not always simultaneously optimizable, and in some cases the computational demands are at odds with the representational aspect. (Humphreys 2004, 95)

In Humphreys's view, a single CM has to be in charge of both representational and computational functions, thus creating a possible tension. My suggestion is that the role of the additional CMs is to ease this tension. The original $\mathrm{CM}$ (here, the quantum $\mathrm{CM}$ ) is the only one in charge of the representation of the original behaviour. The additional two CMs (here, the lattice and statistical), as they are freed from the representation task toward the behaviour, are able to bring additional computational improvements.

Future work could be to look for other cases of multi-layered simulations, outside the field of ITPISR simulations; a candidate could be cellular automata simulations. In the present case study, the bottom CM is statistical: it could be interesting to see if this is always the case.

\section{Acknowledgements}

Many thanks to Anouk Barberousse, Paul Humphreys, Cyrille Imbert, Julie Jebeile, and two anonymous referees of this journal for critical comments or suggestions that helped to improve this paper. This work has been mainly conducted at the IHPST in Paris while I benefited from a doctoral grant from the University of Paris 1 and from the ANR Compuphys project (ANR-08-JCJC-0035-01). 


\section{Bibliography}

Alet, F., and E. S. Sørensen. 2003. "Cluster Monte-Carlo Algorithm for the Quantum Rotor Model." Physical Review E 67: 015701.

Beisbart, C., and J. D. Norton. 2012. "Why Monte Carlo Simulations Are Inferences and Not Experiments." International Studies in the Philosophy of Science 26: 403-422.

Bietenholz, W., R. Brower, S. Chandrasekharan, and U.-J. Wiese. 1997. "Perfect Lattice Topology: The Quantum Rotor as a Test Case." Physics Letters B 407: 283-289.

Boyer, T., W. Bietenholz, and J. Wuilloud. 2007. "Spin Chain Simulations with a Meron Cluster Algorithm." International Journal of Modern Physics C 18: 1497-1511.

Galison, P. 1997. Image and Logic: A Material Culture of Microphysics. Chicago, IL: University of Chicago Press.

Gupta, R. 1998. "Introduction to Lattice QCD." arXiv:hep-lat/9807028.

Gupta, R. 1999. "General Physics Motivations for Numerical Simulations of Quantum Field Theory." Parallel Computing 25: 1199-1215.

Hartmann, S. 1996. "The World as a Process: Simulations in the Natural and Social Sciences." In Modelling and Simulation in the Social Sciences from the Philosophy of Science Point of View, edited by R. Hegselmann, U. Mueller, and K. G. Troitzsch, 77-100. Dordrecht: Kluwer.

Hesse, M. B. 1963. Models and Analogies in Science. London: Sheed and Ward.

Hughes, R. I. G. 1997. "Models and Representation." Philosophy of Science 64 (Proceedings): S325-S336.

Hughes, R. I. G. 1999. "The Ising Model, Computer Simulation, and Universal Physics." In Models as Mediators: Perspectives on Natural and Social Science, edited by M. S. Morgan and M. Morrison, 97-145. Cambridge: Cambridge University Press. Humphreys, P. 1994. "Numerical Experimentation." In Patrick Suppes: Scientific Philosopher, edited by P. Humphreys, vol. 2, 103-118. Dordrecht: Kluwer. Humphreys, P. 2004. Extending Ourselves. New York: Oxford University Press.

Kleinert, H. 2007. Path Integrals in Quantum Mechanics, Statistics, Polymer Physics, and Financial Markets. 4th edition. Singapore: World Scientific.

Lenhard, J. 2007. "Computer Simulation: The Cooperation between Experimenting and 
Modeling." Philosophy of Science 74: 176-194.

Metropolis, N., A. W. Rosenbluth, M. N. Rosenbluth, A. H. Teller, and A. Teller. 1953. "Equation of State Calculations by Fast Computing Machines." Journal of Chemical Physics 21: 1087-1092.

Montvay, I., and G. Münster. 1994. Quantum Fields on a Lattice. Cambridge: Cambridge University Press.

Norton, S., and F. Suppe. 2001. "Why Atmospheric Modeling is Good Science". In Changing the Atmosphere, edited by P. Edwards and C. Miller, 67-105. Cambridge, MA: MIT Press.

Reed, M., and B. Simon. 1975. Methods of Modern Mathematical Physics II. San Diego, CA: Academic Press.

Regt, H. W. de, and D. Dieks. 2005. "A Contextual Approach to Scientific Understanding." Synthese 144: 137-170.

Rothe, H. J. 2005. Lattice Gauge Theories. 3rd edition. Singapore: World Scientific.

Schulman, L. S. 1981. Techniques and Applications of Path Integration. New York: John Wiley \& Sons.

Winsberg, E. 1999. "Sanctioning Models: The Epistemology of Simulation." Science in Context 12: 275-92.

Zinn-Justin, J. 2003. Intégrale de chemin en mécanique quantique. Paris: CNRS éditions. 


\section{Notes}

${ }^{1}$ Cf. for instance Hughes (1999), Winsberg (1999), Norton and Suppe (2001), Humphreys (2004). Hartmann (1996) prefers to speak about 'processes'.

${ }^{2}$ Humphreys's conception seems to be more general than that of others, for instance Hartmann: it 'leave[s] room for simulations of static objects as well' (Humphreys 2004, 108) and Monte-Carlo methods (Humphreys 2004, 106), which Hartmann (1996, 83 and 87-88) excludes.

${ }^{3}$ I do not wish here to diminish the importance of discretization in simulations: a philosophical analysis of it can be found in Lenhard (2007).

${ }^{4}$ Textbook presentations of the quantum rotor can be found in Schulman (1981, § 23.1), Zinn-Justin $(2003, \S 5.6)$, Kleinert $(2007, \S 6.1)$. Papers about an ITPISR simulation of the quantum rotor are for instance Bietenholz et al. (1997) and Boyer, Bietenholz, and Wuilloud (2007).

${ }^{5}$ Path integral was invented by Feynman in the 1940s. Historical elements of the later development can be found in Reed and Simon $(1975,115)$ and Montvay and Münster (1994, 2).

${ }^{6}$ Cf. for example Schulman (1981, ch. 26), Zinn-Justin (2003, ch. 4), Kleinert (2007, $\S 6.2)$.

7The name 'Monte-Carlo' refers to the random character of the casino games. For the origin of the Monte-Carlo method, see Metropolis and Ulam (1949), Galison (1997, chap. 8). Cf. Humphreys (1994), Galison (1997, chap. 8), Beisbart and Norton (2012) for historical and philosophical analyses.

${ }^{8}$ Although the concept of 'model' has been much discussed in the literature, I use it in a wide and uncritical sense here. The vocabulary will be more specific in section 4 .

${ }^{9}$ The paper is actually about a rotor in quantum field theory, but the point about the representation is the same.

${ }^{10}$ For quantum field theory, a table of correspondence similar to Table 1 can be found in Gupta (1998), 22.

${ }^{11} \mathrm{Cf}$. section 2.1. Arguments are made about the quantum rotor, but could easily be generalized for any ITPISR simulation. 
${ }^{12}$ It seems very unlikely that Humphreys or anyone else would like to consider that applying an imaginary time transformation or a reinterpretation is a simulation in itself. If one insisted to say that it amounts to providing a solution to some equation, and thus fits Humphreys's definition, then wouldn't multiplying by -1 just be a simulation as well?

${ }^{13}$ If the label 'theory' may be questioned, it is at least a radical reformulation of the quantum field theory, with a different number of space dimensions for instance.

${ }^{14}$ Note that this proposal could have the following one as a particular case: the statistical CM would belong to the core simulation, and the lattice and quantum models would be added to get the full simulation.

${ }^{15}$ A classical reference on analogical models is Hesse (1963). Note that there exist also analog simulations (e.g. Humphreys 2004, $\S 4.5$ ), in contrast to digital simulations run on computers about which I am only concerned here.

${ }^{16}$ One can also say that the first $\mathrm{CM}$ also represents $B$, but analogically and more indirectly (cf. section 4).

${ }^{17}$ The representational role of a candidate CM may depend on the context and on the use of the simulation by scientists. 